be considered in assessment and intervention in communitydwelling elderly.

\section{AGE-ASSOCIATED FACTORS CONTRIBUTING TO OBSTACLE NEGOTIATION ABILITIES: NOT ALL IS AS EXPECTED}

S. Eyal 2,4, I. Kurz ${ }^{1,4}$, A. Mirelman ${ }^{4,6}$, I. Maidan ${ }^{4}$, N. Gil adi $^{5,6,3}$, J.M. Hausdorff ${ }^{4,2,3}, 1$. Department of Physical Therapy, Faculty of Health Sciences, Ben-Gurion University of the Negev, Beer-Sheva, Israel, 2. Department of Physical Therapy, Sackler Faculty of Medicine, Tel Aviv University, Tel Aviv, Israel, 3. Sagol School of Neuroscience, Tel Aviv University, Tel Aviv, Israel, 4. Center for the Study of Movement, Cognition, and Mobility, Tel Aviv Sourasky Medical Center, Tel Aviv, Israel, 5. Neurological Institute, Tel Aviv Sourasky Medical Center, Tel Aviv, Israel, 6. Department of Neurology, Tel Aviv University, Tel Aviv, Isr ael

Tripping over an obstacle is one of the most common causes of falls among older adults. Using an innovative, computer controlled, obstacle course, we evaluated age-associated changes in the ability to negotiate obstacles and the role of obstacle parameters (e.g., expected vs. unexpected, height and available response time (ART), and subject characteristics (e.g., executive function, gait and balance). Twenty healthy older adults $(77.7 \pm 3.4$ years; $50 \%$ women $)$ and 20 healthy young adults $(29.3 \pm 3.8$ years; $50 \%$ women $)$ underwent cognitive, gait and balance testing before negotiating the computer controlled obstacle course. The primary outcome measure was the ability to successfully negotiate the obstacles (without touching); independent variables included the obstacle height and ART. As hypothesized, the success rate (SR) for all subjects was higher when the obstacle was expected $(99.0 \pm 2.8 \%$; compared to unexpected $66.0 \pm 20.2 \% ; p<0.001)$. With an obstacle height of $25 \mathrm{~mm}$ and an ART of $225 \mathrm{msec}$, SR was lower $(\mathrm{p}<0.001)$ among older adults $(50.0 \pm 40.4 \%)$, as compared to young adults $(100 \pm 0.0 \%)$. For all subjects, the effect of unexpected obstacle height on SR was opposite to our hypothesis; surprisingly, SR was lower when the obstacle height was higher $(\mathrm{p}<0.001)$. For young adults, SR was related to ART $(\mathrm{p}=0.02)$, however, for the older adults, SR was not related to ART. Among the older adults, SR was correlated with stride length $\left(r_{\mathrm{s}}=0.42, \mathrm{p}=0.039\right)$ and Trail Making Test $\mathrm{B}$ $\left(r_{s}=-0.38, p=0.055\right)$. These findings provide new insights into the ability of older adults to successfully negotiate obstacles and help to better understand the mechanisms that underlie this everyday skill.

\section{LIFE-SPACE PREDICTS HEALTHCARE UTILIZATION IN COMMUNITY-DWELLING OLDER ADULTS}

R.E. Kennedy ${ }^{1}$, C. Williams ${ }^{1}$, P. Sawyer ${ }^{1}$, A. Lo ${ }^{3}$, K. Connelly ${ }^{2}$, A. Nassel ${ }^{4}$, C. Brown ${ }^{1}, 1$. Gerontology, Geriatrics, and Palliative Care, The University of Alabama at Birmingham, Birmingham, Alabama, 2. Indiana

University, Bloomington, Indiana, 3. Emergency Medicine, The University of Alabama at Birmingham, Birmingham, Alabama, 4. School of Public Health, The University of Alabama at Birmingham, Birmingham, Alabama

The UAB Life-Space Assessment (LSA) is a validated patient-reported outcome to measure community mobility and social participation. We have previously shown that LSA predicts adverse health outcomes including mortality, nursing home admission, and cognitive decline, but the role of life-space in healthcare utilization among older adults in general is not known. Participants $(\mathrm{N}=400)$ were drawn from the UAB Study of Aging II, a longitudinal study of community-dwelling adults age $\geq 75$ identifying predictors of mobility decline. LSA scores at each monthly follow-up interview were used to predict emergency department (ED) visits and hospitalizations over the next 30 days using generalized estimating equations, adjusting for baseline age, race, gender, education, Charlson comorbidity score, physician visits in the last month, and living alone. Over 35-months of followup, $55.8 \%$ of participants reported at least one ED visit or hospitalization. In multivariable models, a 10-point lower life-space was associated with a $12 \%$ increase in healthcare utilization $(\mathrm{p}<.0001)$. Further investigation is warranted on the utility of life-space scores for predicting unnecessary healthcare utilization and identifying conditions that can be managed in a less intense setting.

\section{FROM HEAD TO TOE, FREQUENCY OF COGNITIVE ACTIVITIES IS ASSOCIATED WITH SHORTER FOOT REACTION TIME}

Y. Cai ${ }^{1}$, S.G. Leveille ${ }^{1,2,3}$, J.M. Hausdorff ${ }^{4}$, J.F. Bean ${ }^{3,5}$, B. Manor ${ }^{3,6}, \mathrm{~T}$. You ${ }^{1}, 1$. University of Massachusetts Boston, Boston, Massachusetts, 2. Beth Israel Deaconess Medical Center, Boston, Massachusetts, 3. Harvard Medical School, Boston, Massachusetts, 4. Tel Aviv University, Tel Aviv, Israel, 5. Veterans Administration Boston Health System, Boston, Massachusetts, 6. Hebrew SeniorLife, Boston, Massachusetts

Cognitively stimulating activities can improve both cognitive and physical function. Foot reaction time, a test of neuromotor performance and a possible risk factor for falls in older adults, might be influenced by participation in cognitively stimulating activities. The aim of this crosssectional study was to examine the association between cognitive leisure activities and foot reaction time. We studied 310 community-dwelling older adults aged 71 to 101 years (mean=84.0y), in the MOBILIZE Boston Study II. Simple foot reaction time (SRT) and choice foot reaction time (CRT) were measured as time to initiation of movement in response to an intermittent light stimulus, seated with feet placed on a gait mat (CIR Systems, Inc., Franklin, NJ). The Cognitive Activity Scale (CAS), assessed frequency of participation in 17 cognitive leisure activities. SRT and CRT ranged from 0.17 to $0.55 \mathrm{~ms}$ (mean $=0.25 \pm 0.06 \mathrm{~ms}$ ), and 0.21 to $0.72 \mathrm{~ms}$ (mean $=0.32 \pm 0.08 \mathrm{~ms}$ ), respectively. Average SRT and CRT were shorter according to higher CAS scores. In multivariable models adjusted for age, sex, race, education, mobility difficulty, peripheral arterial disease, and number of joint pain sites, CAS scores were associated with shorter SRT $(\mathrm{p}=0.02)$ and CRT $(\mathrm{p}=0.01)$. The association persisted but was weaker after further adjusting for gait speed (SRT, $\mathrm{p}=0.05$; CRT, $\mathrm{p}=0.03$ ). Adjustment for vision impairment did not alter the relationship between CAS and reaction time. These results show that participation in cognitively stimulating activities is associated with shorter foot reaction time. Further research is needed to determine whether participation in cognitive activities could reduce fall risk in older adults. 